\title{
ANALYSIS OF A PROPER-MOTION-SELECTED SAMPLE OF STARS IN THE URSA MINOR DWARF SPHEROIDAL GALAXY
}

\author{
PAul B. ESKRIDGE ${ }^{1,2}$ AND ANDREA E. SchweItzer ${ }^{3}$ \\ Received 2001 July 6; accepted 2001 August 13
}

\begin{abstract}
We have studied the stellar population and internal structure of the core of the Ursa Minor dwarf spheroidal galaxy, using a sample of stars selected to be members based on their proper motions. In agreement with previous studies, we find Ursa Minor to be dominated by an old, metal-poor stellar population. A small number of stars with high membership probabilities lie redward of the red giant branch. The brightest $(V \lesssim 18)$ such stars are known to be carbon stars, rather than metal-rich firstascent giants. A number of stars with high membership probabilities lie blueward of the red giant branch and are more luminous than the horizontal branch. We speculate that these are post-horizontal branch stars. There may also be one or two stars in the post-AGB phase. Spectroscopy of the candidate post-HB and post-AGB stars is required to determine their nature. We recover the internal substructure in Ursa Minor that has been noted by several authors in the last 15 years. Using a variety of two- and three-dimensional statistical tests, we conclude that this substructure is statistically significant at the 0.005 level. There is no evidence that the regions of density excess have stellar populations that differ from the main body of Ursa Minor. The crossing time for a typical density excess is only $\sim 5 \times 10^{6} \mathrm{yr}$. They are therefore clearly not due to intermediate age star-forming bursts. We conclude that they are instead due to tidal interactions between the Galaxy and Ursa Minor.
\end{abstract}

Key words: galaxies: dwarf — galaxies: individual (Ursa Minor) — galaxies: stellar content galaxies: structure - Local Group

\section{INTRODUCTION}

The discovery of the Ursa Minor dwarf spheroidal (dSph) galaxy was announced by Wilson (1955), although the first comments on its stellar populations were by Baade (1950). Ursa Minor is one of four dSph satellites of the Galaxy that were identified by direct examination of first epoch Palomar Sky Survey plates (Harrington \& Wilson 1950; Wilson 1955). The first color-magnitude diagram (CMD) was presented by van Agt (1967). These and other early photographic studies led to the conclusion that dSphs were pure Population II systems. Their stellar populations appeared to be the same as those of Galactic globular clusters, but with central stellar densities lower by some 6 orders of magnitude. Several decades later, Ursa Minor remains the only dSph that appears to have a pure Population II stellar population (e.g., Mighell \& Burke 1999; Hernandez, Gilmore, \& Valls-Gabaud 2000).

Hodge (1964) performed the first structural study of Ursa Minor and showed that it is a remarkably elongated system. He found $e=0.55 \pm 0.10$. This compares very well with the result of Irwin \& Hatzidimitriou (1995), who found $e=0.56 \pm 0.05$. Hodge (1964) used star counts on Schmidt plates to fit the stellar distribution of Ursa Minor with an analytic King (1962) model. His results for the core and tidal radii are also consistent with the more modern values of Irwin \& Hatzidimitriou (1995).

The first paper to study the two-dimensional structure of Ursa Minor was Olszewski \& Aaronson (1985). This paper showed evidence for nonaxisymmetric structure in the

\footnotetext{
${ }^{1}$ Department of Astronomy, Ohio State University, Columbus, OH 43210

2 Department of Physics and Astronomy, Minnesota State University, Mankato, MN 56001; paul.eskridge@mnsu.edu.

${ }^{3}$ Little Thompson Observatory, P.O. Box 930, Berthoud, CO 80513; schweitz@frii.com.
}

stellar distribution of Ursa Minor. Both the Fornax (Hodge 1961; Eskridge 1988b; Irwin \& Hatzidimitriou 1995) and Sculptor (Eskridge 1988a; Irwin \& Hatzidimitriou 1995) $\mathrm{dSphs}$ are also known to have nonaxisymmetric structure in their stellar distributions. Recent work (Martínez-Delgado et al. 2001) indicates that a substantial population of extratidal stars exists surrounding Ursa Minor. It is unclear whether the extratidal population is in the form of tidal tails or whether it is an extended diffuse halo. The existence of this extended structure argues that Ursa Minor is, in fact, not in virial equilibrium and may have great impact on our understanding of the halo dwarfs.

In this paper we use the proper-motion-selected sample of Schweitzer (1996; see also Schweitzer, Cudworth, \& Majewski 1997), discussed in $\S 2$, to study the stellar populations and structure of the core of Ursa Minor. In $\S 3$ we test the hypothesis that Ursa Minor is a pure Population II system and seek to either detect any signature of a younger stellar population, or to put a numerical limit on such a contribution. In $\S 4$ we apply a set of statistical tests to the proper-motion-selected sample in order to assess the significance of the substructure noted in the works above. In $\S 5$ we summarize our results and give suggestions for future research. Following Mighell \& Burke (1999), we adopt a distance modulus of $(m-M)_{0}=19.18 \pm 0.12$ and foreground reddening and absorption of $E(B-V)=0.03 \pm 0.01$ and $A_{V}=0.09 \pm 0.03$, giving a linear distance of $69 \pm 4 \mathrm{kpc}$.

\section{DATA ACQUISITION AND REDUCTION}

The data for this study are taken from Schweitzer (1996). That work provided membership probabilities based on proper motions for stars brighter than $V \approx 20.5$ (slightly fainter than the horizontal branch) within $\sim 23^{\prime}$ of the adopted centroid of Ursa Minor: $\alpha_{2000.0}=15^{\mathrm{h}} 08^{\mathrm{m}} .8$, $\delta_{2000.0}=+67^{\circ} 12^{\prime}$. The area surveyed is larger than the 
Ursa Minor core $\left(r_{c}=15^{\prime} .8 \pm 1\right.$ 1'2, Irwin \& Hatzidimitriou 1995). The catalog has a total of 1533 stars. Of these, 1060 have a nonzero membership probability. The membershipweighted sample is 867.5 stars.

\section{STELLAR POPULATIONS}

Figure 1 shows the $V-(B-V)$ CMD for the full sample. The red giant branch (RGB) is visible from $V \approx 17$, $(B-V) \approx 1.2$ to $V \approx 20,(B-V) \approx 0.7$. The horizontal branch (HB) is also visible and, as is well known (e.g., van Agt 1967; Olszewski \& Aaronson 1985; Mighell \& Burke 1999), dominated by blue HB (BHB) stars. There are also a large number of RR Lyrae variables in the sample. However, the CMD is contaminated by a large number of foreground stars. We can use our membership probabilities to produce a much cleaner CMD, shown in Figure 2. This figure compares well with Figure 5 of Cudworth, Olszewski, \& Schommer (1986), which was based on an earlier propermotion solution with a shorter time baseline. In Figure 2 the size of the symbol corresponds to the membership probability $(P)$, with the smallest dots having $P<0.1$ and the largest circles having $P>0.9$. We note that the RGB is now a very well-defined feature and that there is evidence for an asymptotic giant branch (AGB) as well. There are a number of stars with high membership probabilities in unusual places of the CMD.

In region $\mathrm{I}$, there are three stars with $P>0.5$ that are substantially brighter and bluer than the RGB tip. The joint probability that all three of these stars are nonmembers is only $\approx 0.007$. We speculate that one or more of them may

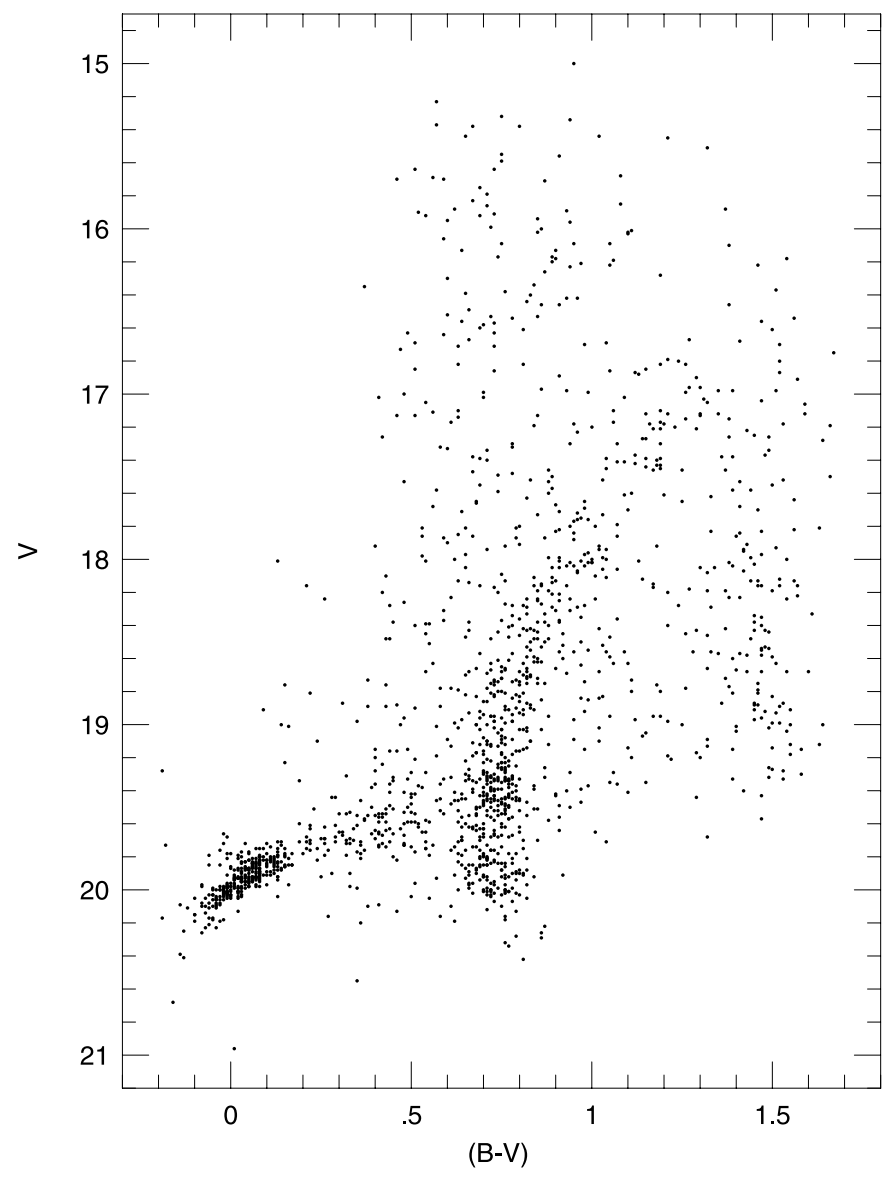

FIG. 1. $-V-(B-V) \mathrm{CMD}$ of the full Ursa Minor sample be evolving across the Hertzsprung-Russell diagram toward the planetary nebula stage. None of them appear to have been studied spectroscopically. This seems like a project well worth undertaking.

In region II, there are a dozen stars with $P>0.5$. Although one of these stars (shown with a cross in Fig. 2, left) is known to be a nonmember (Hargreaves et al. 1994), the joint probability that none of them are members is less than $10^{-13}$. The members in this region would be post-HB or AGB stars.

Finally, in region III, there are 17 stars with $P>0.5$. Eight of these are velocity members based on the spectroscopy of Hargreaves et al. (1994), Armandroff, Olszewski, \& Pryor (1995), and Olszewski, Aaronson, \& Hill (1995). Two are velocity nonmembers according to Shetrone, Côté, \& Stetson (2001b). The other seven have not been observed spectroscopically. The joint probability that none of these seven stars are members is $P \approx 5 \times 10^{-5}$. Member stars in this region of the CMD are candidate carbon stars. Indeed, of the eight confirmed members, five are known to be carbon stars (Armandroff et al. 1995; Shetrone et al. 2001b). One is a red giant (Shetrone et al. 2001b), very close to, but slightly redward of, the RGB tip. In Figure 2 (left) the nonmembers are shown with crosses, the carbon stars with squares, and the red RGB tip star with a triangle.

In Table 1 we give the positions, magnitudes, and colors of the stars in all three regions of the CMD as an aid for future spectroscopic observations. We note those stars that are known to be velocity members based on the spectroscopy of Hargreaves et al. (1994), Armandroff et al. (1995), Olszewski et al. (1995), and Shetrone et al. (2001b).

In Figure 2 (right) we show the ridge lines for the RGBs of a set of globular clusters, overlaid on the Ursa Minor CMD. We use the globular clusters M68 (Walker 1994), M55 (Lee 1977), NGC 6752 (Cannon \& Stobie 1973), NGC 362 (Harris 1982), and 47 Tuc (Hesser et al. 1987), with minor corrections as given in Da Costa et al. (1996) and shifted to account for the distance and reddening of Ursa Minor. In Figure 3 we show the probability-weighted CMD from Figure 2, along with sets of theoretical isochrones from Girardi et al. (2000). As with the giant branch ridge lines, we have shifted the isochrones to account for the distance and reddening of Ursa Minor. We show a set of 14 Gyr isochrones for a range of abundances in Figure 3 (left) and a set of isochrones with $[\mathrm{Fe} / \mathrm{H}]=-1.7$ for a range of ages in Figure 3 (right). As shown by extensive previous work (Shetrone, Côté \& Sargent 2001a and references therein), Ursa Minor is dominated by an old, metal-poor stellar population. As is clear from Figure 2 (right) and Figure 3 (left), there are proper-motion members of Ursa Minor that fall on the RGB loci of old, comparatively metal-rich stellar populations $([\mathrm{Fe} / \mathrm{H}] \approx-0.7)$. Recent spectroscopy of some of the brighter stars in this region of the CMD shows them to be either nonmembers or carbon stars (Shetrone et al. 2001b). At the moment, there is no observational support for the existence of an old, but metalrich, stellar population in Ursa Minor.

Figure 4 shows the $B$ - and $V$-band luminosity functions (LFs) of Ursa Minor, incorporating our membership probabilities. The error bands are simply root- $N$ errors of the unweighted number of stars. That is, any star with a nonzero membership probability adds one to the sum for error calculations, but adds only $P_{i}$ to the LF. We applied a Sobel filter (Madore \& Freedman 1995; Lee, Freedman, \& Madore 1993) in order to search for structure in the LFs. 
TABLE 1

CMD OUTLIERS WITH $P>0.5$

\begin{tabular}{|c|c|c|c|c|c|c|c|c|c|c|}
\hline ID & $V$ & $(B-V)$ & $P$ & $\begin{array}{c}X \\
(\operatorname{arcsec})\end{array}$ & $\begin{array}{c}Y \\
(\operatorname{arcsec})\end{array}$ & $\begin{array}{l}\text { R.A. } \\
\text { (J2000.0) }\end{array}$ & $\begin{array}{l}\text { Decl. } \\
(\text { J2000.0) }\end{array}$ & $\operatorname{COS}^{a}$ & Irwin $^{b}$ & Vel. Mem. ${ }^{\mathrm{c}}$ \\
\hline 746 & 16.17 & 0.74 & 0.51 & -3.3 & 745.2 & 150851.60 & 672511.5 & $\ldots$ & $\ldots$ & $\ldots$ \\
\hline 783 & 15.86 & 0.71 & 0.96 & -564.4 & 465.5 & 150714.50 & 672029.9 & $\ldots$ & $\ldots$ & $\ldots$ \\
\hline 1406 & 16.09 & 1.05 & 0.67 & -513.2 & -99.4 & 150723.94 & 671105.4 & $\ldots$ & $\ldots$ & $\ldots$ \\
\hline 177 & 18.48 & 0.43 & 0.96 & 797.5 & 915.4 & 151110.90 & 672758.0 & $\ldots$ & $\ldots$ & $\ldots$ \\
\hline 253 & 18.76 & 0.15 & 0.80 & -260.3 & 1300.1 & 150806.68 & 673426.0 & $\ldots$ & $\ldots$ & $\ldots$ \\
\hline 296 & 18.81 & 0.22 & 0.96 & -977.9 & 947.1 & 150602.00 & 672827.8 & $\ldots$ & $\ldots$ & $\ldots$ \\
\hline 395 & 19.23 & 0.15 & 0.91 & 23.9 & 1202.8 & 150856.22 & 665243.5 & $\ldots$ & $\ldots$ & $\ldots$ \\
\hline 690 & 18.16 & 0.21 & 0.91 & 378.3 & 907.0 & 150957.97 & 672752.4 & $\ldots$ & $\ldots$ & $\ldots$ \\
\hline 916 & 19.34 & 0.19 & 0.97 & -676.6 & -606.4 & 150656.53 & 670237.3 & $\ldots$ & $\ldots$ & $\ldots$ \\
\hline 949 & 19.00 & 0.14 & 0.88 & -644.3 & -801.3 & 150702.28 & 665922.7 & $\ldots$ & $\ldots$ & $\ldots$ \\
\hline 1089 & 17.92 & 0.40 & 0.91 & 673.5 & 21.6 & 151048.12 & 671305.2 & $\ldots$ & $\ldots$ & $\ldots$ \\
\hline 1259 & 19.01 & 0.16 & 0.93 & 444.3 & 593.8 & 151009.17 & 672238.9 & $\ldots$ & $\ldots$ & $\ldots$ \\
\hline 1537 & 17.53 & 0.88 & 0.84 & -113.9 & -496.0 & 150832.68 & 670430.2 & 442 & 37482 & No \\
\hline 1600 & 19.10 & 0.24 & 0.98 & 63.8 & -157.9 & 150903.14 & 671008.4 & $\ldots$ & $\ldots$ & $\ldots$ \\
\hline 1710 & 18.45 & 0.54 & 0.83 & 136.6 & 341.1 & 150915.78 & 671827.2 & $\ldots$ & $\ldots$ & $\ldots$ \\
\hline 152 & 17.62 & 1.33 & 0.74 & 1055.7 & 748.7 & 151155.47 & 672508.5 & $\ldots$ & 30614 & Yes, C \\
\hline 251 & 18.07 & 1.42 & 0.91 & -445.2 & 1315.8 & 150734.37 & 673440.9 & $\ldots$ & $\ldots$ & No \\
\hline 368 & 19.29 & 1.06 & 0.52 & -431.1 & -998.1 & 150738.81 & 665607.1 & $\ldots$ & $\ldots$ & $\ldots$ \\
\hline 440 & 19.13 & 1.32 & 0.54 & 187.6 & -809.1 & 150924.17 & 665916.9 & $\ldots$ & $\ldots$ & $\ldots$ \\
\hline 505 & 18.95 & 1.17 & 0.56 & 1052.9 & -8.4 & 151153.38 & 671231.5 & $\ldots$ & $\ldots$ & $\ldots$ \\
\hline 536 & 17.58 & 1.39 & 0.88 & 950.2 & 326.1 & 151136.35 & $\begin{array}{lll}67 & 18 & 07.1\end{array}$ & $\ldots$ & 32961 & Yes, C \\
\hline 829 & 17.94 & 1.42 & 0.75 & -759.9 & -94.6 & 150641.52 & 671108.3 & $\ldots$ & $\ldots$ & No \\
\hline 979 & 18.45 & 1.26 & 0.96 & -160.8 & -919.4 & 150824.79 & 665726.8 & $\ldots$ & $\ldots$ & $\ldots$ \\
\hline 1008 & 19.65 & 1.01 & 0.81 & 221.2 & -552.0 & 150930.01 & 670334.0 & $\ldots$ & $\ldots$ & $\ldots$ \\
\hline 1128 & 17.15 & 1.38 & 0.66 & 384.9 & 214.1 & 150958.59 & 671619.5 & 82 & 33521 & Yes \\
\hline 1167 & 18.05 & 1.34 & 0.87 & 574.7 & 381.7 & 151031.54 & 671906.1 & 122 & 32613 & Yes, C \\
\hline 1363 & 18.73 & 1.11 & 0.78 & -303.9 & 162.8 & 150759.77 & 671528.6 & $\ldots$ & $\ldots$ & $\ldots$ \\
\hline 1545 & 17.26 & 1.38 & 0.66 & -96.5 & -545.2 & 150835.67 & 670341.1 & $\ldots$ & 37759 & Yes, C \\
\hline 1677 & 16.54 & 1.56 & 0.98 & 125.1 & 166.8 & 150913.74 & 671533.0 & 60 & 33767 & Yes \\
\hline 1721 & 18.48 & 1.44 & 0.67 & -65.3 & 286.7 & 150840.90 & 671733.0 & $\ldots$ & $\ldots$ & $\ldots$ \\
\hline 1846 & 16.91 & 1.57 & 0.99 & -145.6 & -158.9 & 150827.16 & 671007.3 & 347 & 35606 & Yes, R \\
\hline 1859 & 17.92 & 1.18 & 0.94 & -251.5 & -204.6 & 150808.98 & 670921.3 & $\ldots$ & 35869 & Yes, C \\
\hline
\end{tabular}

NoTE.-Units of right ascension are hours, minutes, and seconds, and units of declination are degrees, arcminutes, and arcseconds.

${ }^{a}$ Numbers from Cudworth et al. (1986).

b Numbers from M. Irwin 2001, private communication.

c Velocity membership status from Hargreaves et al. 1994, Armandroff et al. 1995, Olszewski et al. 1995, or Shetrone et al. 2001. "C": carbon stars according to Armandroff et al. 1995, or Shetrone et al.2001. "R": RGB star according to Shetrone et al. 2001.

We show an example of the binned differential $V$-band LF and the resulting Sobel-filter output in Figure 5. The RGB tip is at $V \approx 16.4$ (or $M_{V} \approx-2.9$ ). There is an apparent gap at $V \approx 17.5$, and the red HB stars cause a strong feature at about $V \approx 19$. No other features are obvious in the Sobelfilter output. The gap at $V \approx 17.5$ is apparent in the CMD. But there is no reason to expect such a gap at $M_{V} \approx-1.9$ in an old, metal-poor RGB. To investigate this further, we constructed a pure RGB $V$-band LF. We did this by selecting stars in the unnumbered region of Figure 2 (left) down to $V=19$. In Figure 6 we show the logarithmic integrated $V$-band RGB LF (solid line), along with Poisson error bars, computed as above (dotted lines), and a generic RGB LF with a slope of 0.6 (dashed line). This shows that the apparent gap at $V \approx 17.5$ is consistent with being a statistical fluctuation at about the $1 \sigma$ level.

\section{TESTS FOR SUBSTRUCTURE}

\subsection{Background}

Evidence for nonaxisymmetric structure in Ursa Minor was first noted by Olszewski \& Aaronson (1985). However, this was based on an analysis of the distribution of $\approx 3000$ stars in a small $\left(\approx 3^{\prime} \times 5^{\prime}\right)$ field. Subsequent studies by Irwin $\&$ Hatzidimitriou (1995) and Kleyna et al. (1998) reinforce the notion that there is nonaxisymmetric clumping in the distribution of stars in Ursa Minor, but none of these three studies has been able to make a firm statement on the statistical significance of these structures.

Demers et al. (1995) rediscovered one of Olszewski \& Aaronson's (1985) stellar clumps with their larger format CCD data, but they did not realize this at the time for an accumulation of reasons given in Battinelli \& Demers (1999). Battinelli \& Demers (1999) use deep, but very small format, $H S T /$ WFPC2 data to argue that the density excesses indicated by earlier work are actually due to a ring with a central depression. This is a truly remarkable suggestion and warrants further study by any other means possible.

Our data are not as deep as the Olszewski \& Aaronson (1985) CCD data and cover a smaller field than the Irwin \& Hatzidimitriou (1995) Schmidt plate scans. However, our data combine a moderate field of view (roughly $40^{\prime} \times 40^{\prime}$ ) with a moderate depth $\left(V_{\lim } \approx 20.5\right)$ and with propermotion-based membership probabilities. Thus the thorny problem of foreground subtraction is much less of an issue 

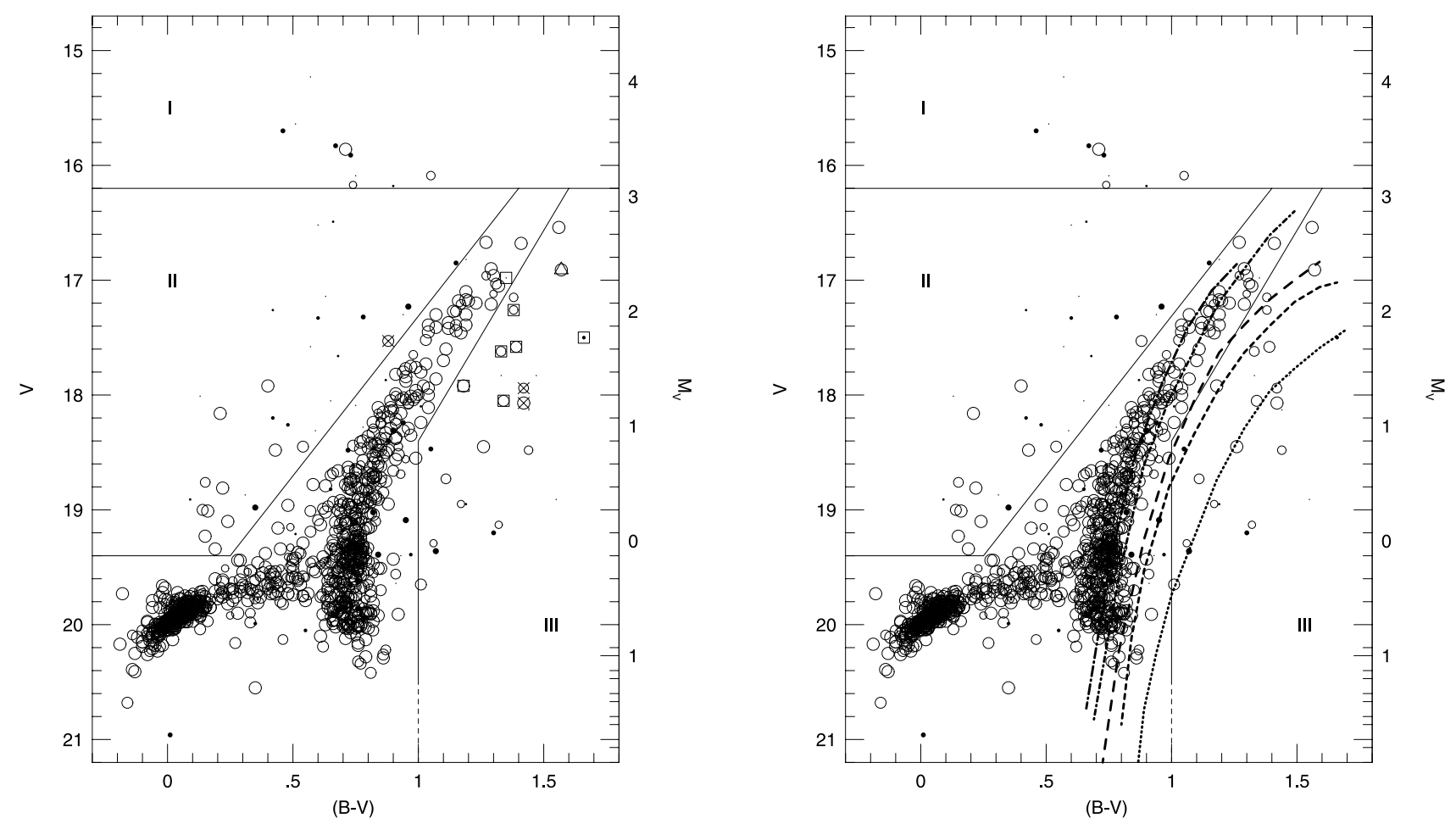

FIG. 2. $-V-(B-V)$ CMD incorporating probability weights. The smallest solid dots have membership probabilities of $P<0.1$. The largest open circles have membership probabilities of $P>0.9$. Stars with $P>0.5$ are shown as open symbols. The solid lines demark regions of the CMD, labelled with roman numerals I-III, from which the stars listed in Table 1 were drawn. Left: The squares show spectroscopically confirmed member carbon stars; the triangle shows the spectroscopically confirmed member RGB star; the crosses show the spectroscopically confirmed nonmembers. Right: The curves show globular cluster RGB ridge lines taken from the literature. The clusters shown are 47 Tuc (dotted line), NGC 362 (short dashed line), NGC 6752 (long dashed line), M55 (dot-short dash line), and M 68 (dot-long dash line).
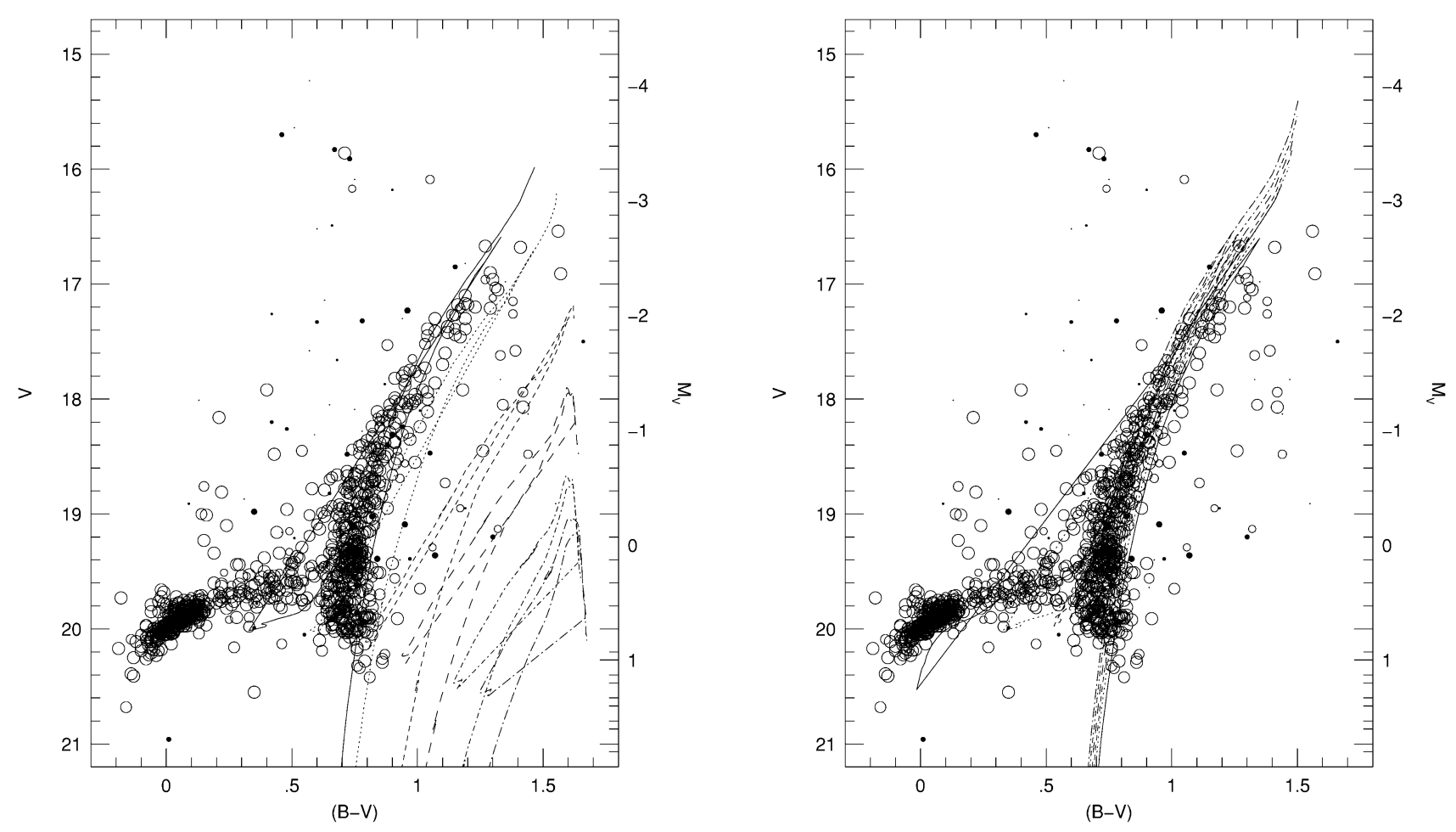

FIG. 3. $-V-(B-V)$ CMD from Fig. 2, with isochrones from Girardi et al. (2000) overlaid. Right: $14 \mathrm{Gyr}$ isochrones with $[\mathrm{Fe} / \mathrm{H}]=-1.7$ (solid line), -1.3 (dotted line), -0.7 (short-dashed line), -0.4 (long-dashed line), 0 (short-dashed-dotted line) and +0.2 (long-dashed-dotted line). Right: [Fe/H] $=-1.7$ isochrones with ages of $18 \mathrm{Gyr}$ (solid line), $14 \mathrm{Gyr}$ (dotted line), $11 \mathrm{Gyr}$ (short-dashed line), $9 \mathrm{Gyr}$ (long-dashed line), $7 \mathrm{Gyr}$ (short-dashed-dotted line), and 5.6 Gyr (long-dashed-dotted line). 

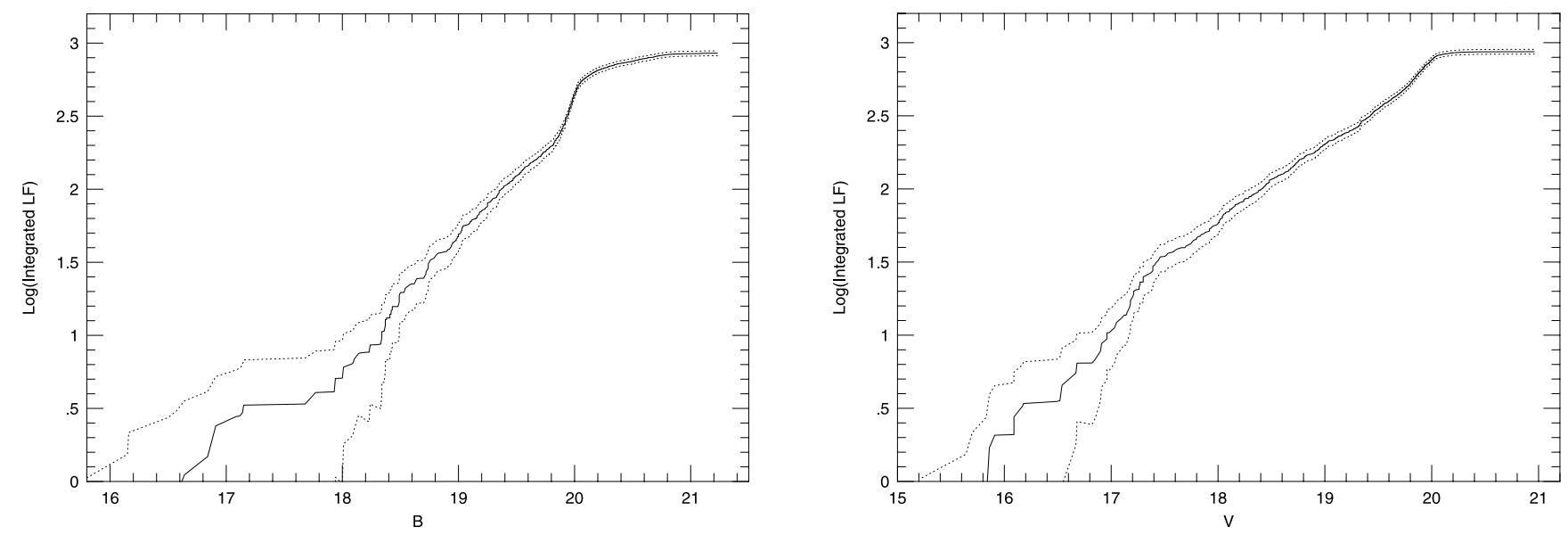

FIG. 4.-Logarithmic probability-weighted integrated LF of Ursa Minor. The solid lines are the LFs, and the dashed lines represent root- $N$ error bounds of the unweighted number of stars. Left: $B$-band LF. Right: $V$-band LF.
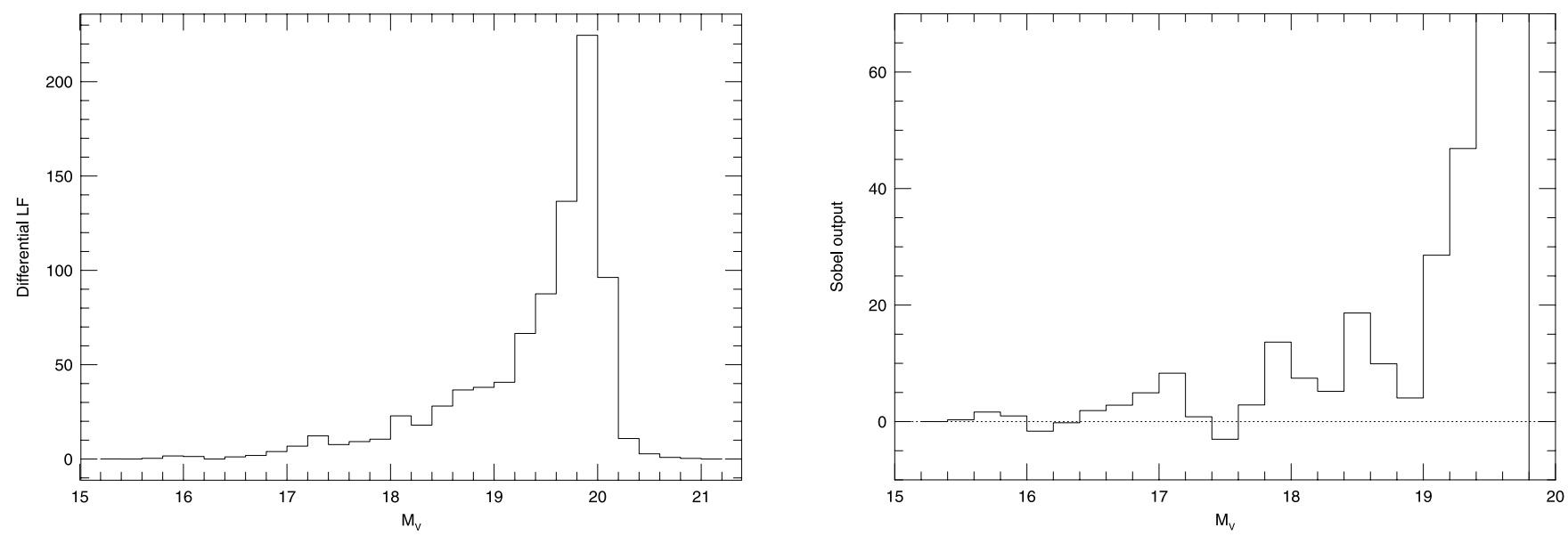

FIG. 5.-Left: Differential $V$-band LF with $0.2 \mathrm{mag}$ binning. Right: Sobel-filter output of the data shown in left panel.

for our data than for any of the previous data sets used for structural studies.

In Figure 7 (top left), we show a contour plot of the probability-weighted stellar density of our sample. Figure 7

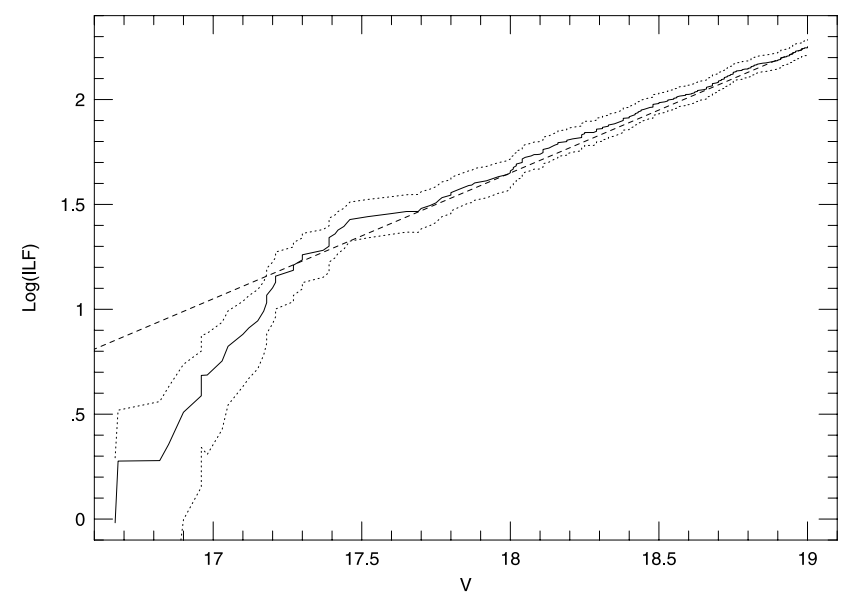

FIG. 6.-Logarithmic $V$-band integrated LF of the RGB of Ursa Minor. The solid line is the LF, the dashed lines represent root- $N$ error bounds of the unweighted number of stars, and the dashed line is a model LF with a power-law index of 0.6. (top right) shows a gray-scale map of the density of nonmembers. We applied a spatial median filter of $9^{\prime}$ to the probability-weighted membership image. The filter box size is roughly the minor-axis core radius of Ursa Minor as reported by Irwin \& Hatzidimitriou (1995). We experimented with a range of filter kernels and found the $9^{\prime}$ to be the best at removing the large-scale structure of Ursa Minor without filtering out the regions we are interested in. After generating the filtered image, we then subtracted it from the raw image, and we show the result in Figure 7 (bottom). Density excesses on either side of the centroid are now obvious in the median-filter subtracted data. We give the positions of these density excesses in Table 2 . The essential question that no previous study has resolved is the following: Are these substructures statistically significant?

\subsection{Analysis}

In many respects, this problem is similar to that of searching for substructure in clusters of galaxies. The significant distinction is that one has position and radial velocity information for galaxy cluster studies, while we have position and proper-motion information. Our problem can be made dimensionally equivalent to that of galaxy cluster studies by taking membership probability as our third measurement.

Pinkney et al. (1996) compile a set of one-, two-, and three-dimensional tests for substructure of sparse data that 

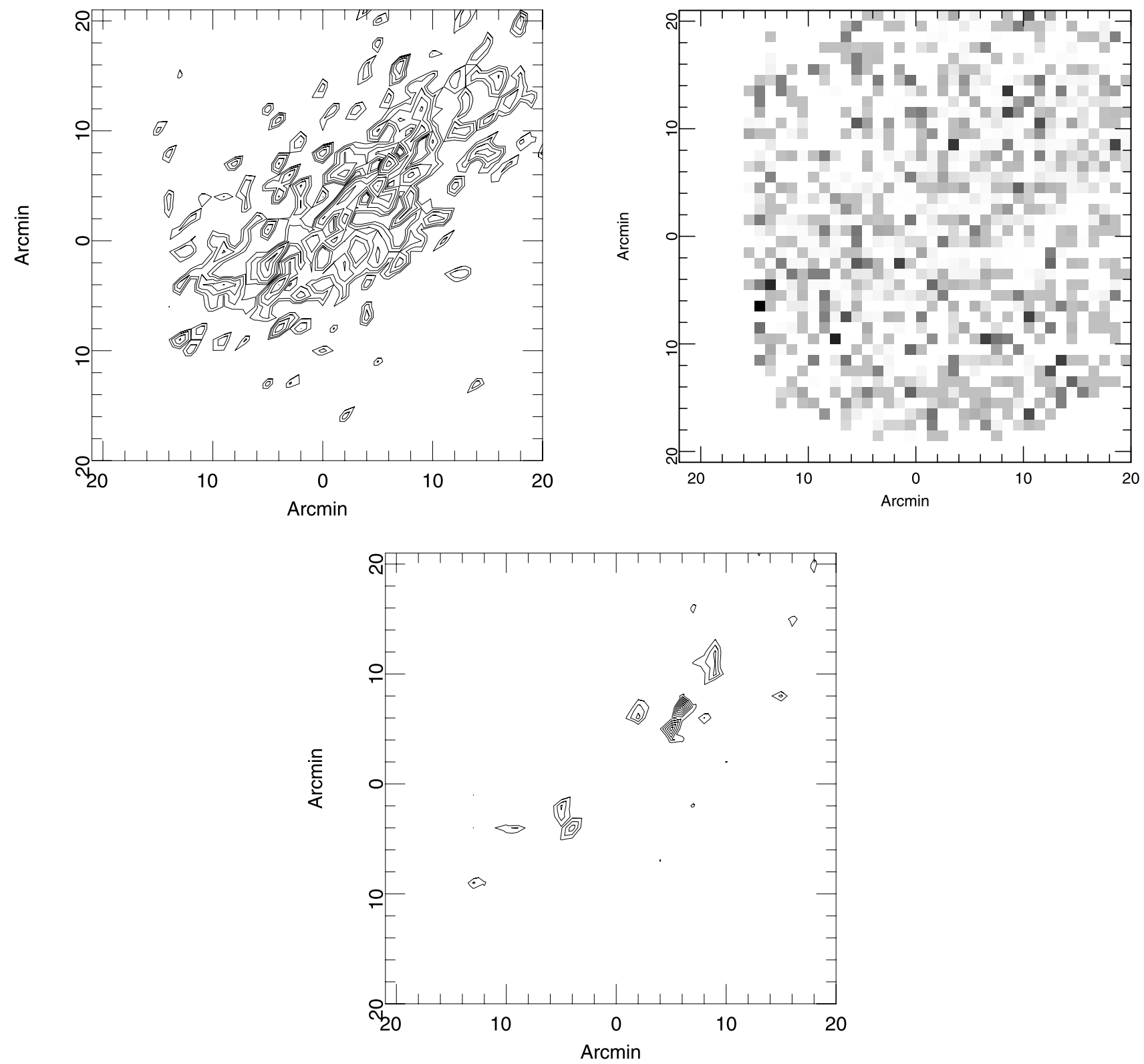

FIG. 7.-Density maps of stars in the Ursa Minor sample. The origin is the adopted center of Ursa Minor. North is up, east to the left. Top left: Contour plot of the probability-weighted density of Ursa Minor stars binned into a $1^{\prime} \times 1^{\prime}$ image. Top right: Gray-scale plot of the nonmembers at the same resolution. Bottom: Contour plot of the residuals of the membership-weighted array minus a median-filtered version of the array, with a 9' filter box.

TABLE 2

\begin{tabular}{|c|c|c|}
\hline Number & $\begin{array}{l}\text { R.A. } \\
\text { (J2000.0) }\end{array}$ & $\begin{array}{l}\text { Decl. } \\
\text { (J2000.0) }\end{array}$ \\
\hline $1 .$. & 150800 & 670630 \\
\hline $2 \ldots \ldots \ldots$ & 150830 & 671020 \\
\hline & 150840 & 671230 \\
\hline $4 \ldots \ldots \ldots$ & 150910 & 671140 \\
\hline $5 \ldots \ldots \ldots$ & 151010 & 672150 \\
\hline $6 \ldots \ldots$ & 151030 & 671950 \\
\hline
\end{tabular}

NOTE.-Units of right ascension are hours, minutes, and seconds, and units of declination are degrees, arcminutes, and arcseconds. they have applied to galaxy cluster studies. We have applied a subset of these tests to our sample, using software kindly provided by J. Pinkney. Specifically, we use the $\beta$, AST, and Lee two-dimensional tests from the two-dimensional substructure tests and the Lee three-dimensional, $\Delta, \alpha, \alpha_{\text {var }}$, and $\epsilon$ tests from the three-dimensional substructure tests. We do not use the one-dimensional tests, as they search only for substructure in the third variable. Nor do we use the Fourier elongation test, as we already know that Ursa Minor is elongated.

In Table 3, we show the results of the two-dimensional and three-dimensional tests. The analysis was done twice; once using the full data set $(N=1060)$ and once using a subsample of all objects with $P$ within $3 \sigma$ of $\bar{P}(N=890)$. In 
TABLE 3

Substructure Test Results

\begin{tabular}{clcc}
\hline \hline \multicolumn{1}{c}{ Sample } & \multicolumn{1}{c}{ Test } & Statistic & Probability \\
\hline Full sample $(N=1060) \ldots .$. & $\beta$ & 11.3 & 0.000 \\
& AST & 13.5 & 0.000 \\
& Lee two-dim. & 1.758 & 0.000 \\
& Lee three-dim. & 1.339 & 0.000 \\
& $\Delta$ & 10637 & 1.000 \\
& $\alpha$ & 181.188 & 0.898 \\
Subsample $(N=890) \ldots . . .$. & $\beta$ & 182.320 & 0.846 \\
& $\alpha_{\text {var }}$ & $1.57 \mathrm{E} 11$ & 0.427 \\
& $\epsilon$ & 8.2 & 0.006 \\
& AST & 11.9 & 0.000 \\
& Lee two-dim. & 1.820 & 0.000 \\
& Lee three-dim. & 1.369 & 0.000 \\
& $\Delta$ & 3726 & 0.409 \\
& $\alpha$ & 203.355 & 0.756 \\
& $\alpha_{\text {var }}$ & 203.086 & 0.756 \\
& $\epsilon$ & $6.96 \mathrm{E} 10$ & 0.002 \\
\hline
\end{tabular}

practice, the 170 stars removed by the clipping procedure have $P<0.63$. All three two-dimensional tests return highly significant results for substructure using both the clipped and unclipped samples. Among the three-dimensional tests, the Lee three-dimensional test returns highly significant results for substructure using both the clipped and unclipped samples. The $\Delta, \alpha$, and $\alpha_{\mathrm{var}}$ tests consistently give no positive results for substructure, and the $\epsilon$ test argues for substructure only when applied to the clipped data set.

The $\beta$ test (West, Oemler, \& Dekel 1988) returns evidence for statistically significant subclustering in Ursa Minor on both the clipped and unclipped samples. The $\beta$ statistic is a measure of deviation from mirror symmetry. Pinkney et al. (1996) note that the $\beta$ statistic does not measure deviation from circular symmetry, so our result is not simply due to the known elongation of Ursa Minor. Rather, this test argues that the distribution of stars about the galaxy center is clumpy, with the clumps not being distributed according to mirror symmetry.

The angular separation test, or AST (West et al. 1988), also returns evidence for statistically significant subclustering in Ursa Minor on both the clipped and unclipped samples. This test measures the excess of small-angle separations above that expected due to Poisson noise. West et al. (1988) examined the behavior of the AST on elongated structures and found that false positives did not occur for ellipticities up to $e \sim 0.5$. As Ursa Minor has $e=0.56 \pm 0.05$ (Irwin \& Hatzidimitriou 1995), this is unlikely to be the cause of the positive signal we see for the AST.

The Lee two-dimensional test (Lee 1979) also returns evidence for statistically significant subclustering in Ursa Minor on both the clipped and unclipped samples. This test measures the significance of separating the data set into two subclusters. As with the $\beta$ test, an elongated but smooth distribution will not cause the Lee statistic to report a false positive.

The Lee three-dimensional test (Fitchett \& Webster 1987) is an extension of the Lee two-dimensional test that incorporates variation in the third dimension. We note that if the spatial subclusters have essentially the same distribution in the third variable (membership probability, in the case of our Ursa Minor data), the Lee three-dimensional test should give the same result as the Lee two-dimensional test. This is the result we find for our Ursa Minor data.

Of the other three-dimensional tests, the $\Delta, \alpha$, and $\alpha_{\text {var }}$ tests consistently give no positive results for substructure. The $\Delta$ statistic (Dressler \& Shectman 1988) looks for significant variations in the third dimension for spatial clumps. As our third dimension is membership probability, the null result we obtain simply says that the spatial subclusters (if any) are part of Ursa Minor. That is, the stars in the spatial subclusters have the same distribution of membership probability as do the rest of the Ursa Minor stars. The $\alpha$ (West \& Bothun 1990) and $\alpha_{\mathrm{var}}$ (Pinkney et al. 1996) tests both measure the dispersions of the third dimension for spatial clusters, and thus, like the $\Delta$ statistic, they would not be expected to show significant subclustering in the present case.

This brings us to the $\epsilon$ test (Bird 1993). In this context, $\epsilon$ is a measure of the average number of stars per nearest neighbor group. Paradoxically, this test gives strong evidence for subclustering on the clipped data set, but no evidence for subclustering on the unclipped data set. This appears to be due to the diluting effects of the low membership probability stars on the test statistic (see discussion in Pinkney et al. 1996). When only the high membership probability stars are retained, the dispersion in membership probability for any spatial subcluster will be much smaller, leading to a more significant result for the $\epsilon$ test.

The essential result of this set of tests is that we confirm the previous evidence for substructure in Ursa Minor and can now quantify the existence of this substructure with a statistical confidence of greater than 0.995 for all well-posed tests of that structure. The distribution of member stars in Ursa Minor is not well described by a smooth distribution. In $\S 5$, we discuss the implications of this for the evolution of Ursa Minor.

\section{SUMMARY AND DISCUSSION}

We have analyzed a proper-motion-selected sample of stars in the Ursa Minor dwarf spheroidal galaxy in order to study the stellar populations and structure of this object. The data are taken from Schweitzer (1996) and include stars within $\sim 23^{\prime}$ of the Ursa Minor centroid, down to a limiting magnitude of $V \approx 20.5$. The membership-weighted sample is 867.5 stars from a total sample of 1533 .

The CMD for our sample compares very well with previous bright-star CMDs for Ursa Minor (van Agt 1967; Cudworth et al. 1986), showing the dominant old, metalpoor stellar population. Comparison with globular cluster $\mathrm{RGB}$ ridge lines and model isochrones indicates a metallicity of $[\mathrm{Fe} / \mathrm{H}] \approx-1.8$ to -2 , consistent with the spectroscopic result of $[\mathrm{Fe} / \mathrm{H}]=-1.90 \pm 0.11$ from Shetrone et al. (2001a). There are a number of high-probability members in unusual parts of the CMD. We provide position and magnitude data for these stars as an aid for future spectroscopic observations. The $B$ - and $V$-band LFs of our sample are consistent with a single, dominant, old, metalpoor stellar population.

We have applied a number of tests for substructure to our sample in order to evaluate the statistical significance of the well-known clumps of stars in Ursa Minor (Olszewski \& Aaronson 1985; Irwin \& Hatzidimitriou 1995; Demers et al. 1995; Kleyna et al. 1998; Battinelli \& Demers 1999). These tests are drawn from the study of Pinkney et al. (1996), who compiled a set of one-, two-, and three- 
dimensional tests for substructure in sparse data for galaxy cluster studies. When the detailed assumptions and behaviour of these tests are taken into account, we conclude that the hypothesis that Ursa Minor has statistically significant substructure is strongly supported (see Table 3 ). We also employed a test devised by Mighell (2001) to search for structure in sparse two-dimensional Poisson-distributed data. The test is a modification of the $\chi^{2}$ statistic. Given a model, the test reports the significance of the deviation of the data from the model. As the test is well-defined for integers, we have used the sample of all stars with $P \geq 0.9$ of being members of Ursa Minor. Our model is a simple elliptical profile fit to these data, with ellipticity and position angle as free parameters. The test rejects the null hypothesis that the data follow the model at the $95 \%$ confidence level. Thus, this test is consistent with the results from the clustering statistics discussed in $\S 4$ and indicates that the distribution of stars in Ursa Minor is not well fitted with a smooth model.

We do not see evidence of the structure reported by Battinelli \& Demers (1999). The lack of agreement between our study and theirs is likely due to the very different strengths and weaknesses of the data for the two studies. The Battinelli \& Demers (1999) study is based on very deep data covering a very small field of view (a single WFPC2 frame), whereas our data are very shallow, but cover a much larger field of view. A proper test of the results of Battinelli \& Demers (1999) would require much deeper wide-field data than we have at our disposal.

The angular size of the lumps is roughly $3^{\prime}$ (see Fig. 7, bottom). This is about $20 \%$ of the size of the core radius. The measured velocity dispersion of Ursa Minor is roughly 10 $\mathrm{km} \mathrm{s}^{-1}\left(10.4 \pm 0.9 \mathrm{~km} \mathrm{~s}^{-1}\right.$ according to Armandroff et al. 1995). For our adopted distance of $69 \pm 4 \mathrm{kpc}$, this implies a lump crossing time of only $\sim 5 \times 10^{6} \mathrm{yr}$. However, neither the CMD, nor the $V$-band LF of stars in the lumps shows any evidence for differences in the stellar populations of the lumps and the bulk of Ursa Minor. The lumps are clearly not due to young (or even intermediate-age) star formation events. We speculate that they are instead due to tidal stretching from the gravitational interaction between the Galaxy and Ursa Minor. Features like the Ursa Minor lumps appear in some numerical simulations of such interactions (e.g., Kroupa 1997; Klessen \& Kroupa 1998). However, the evidence for such dynamically induced structure and for extratidal stars associated with Ursa Minor (Martínez-Delgado et al. 2001) is unlikely to provide an escape for the large mass to light ratio for Ursa Minor that is implied by its velocity dispersion, as numerical studies indicate that the observed velocity dispersions of disrupting dwarfs do not significantly exceed their viral values until just before their complete dispersal (e.g., Oh, Lin, \& Aarseth 1995; Piatek \& Pryor 1995).
It would be very interesting to obtain spectra of the remaining high-probability member stars in the outlying regions of the CMD. The stars in region I are bright enough $(V<16.2)$ that spectroscopy with a 4 m-class telescope should be able to determine their membership and their nature. All the high-probability stars in regions II and III that have not yet been observed spectroscopically are fainter than $V \approx 18$ and thus become difficult targets for 4 $\mathrm{m}$-class spectroscopy. Determining the membership and nature of these stars would be an excellent project for the HET, the upgraded MMT, or the Keck telescope, or, in the future, for the Gemini telescope. Very deep photometry of Ursa Minor, both in the lumps and in the general field, would provide a much better probe of the stellar populations than the data currently available. The main problem with the WFPC2 data is that the field of view is so small. Observations of a number of fields in the halo dwarf spheroidals with the MOSAIC imager on the Mayall and Blanco $4 \mathrm{~m}$ telescopes and with the forthcoming Advanced Camera for Surveys, will put our understanding of the details of the star formation histories of the halo dwarfs on a much better observational footing than is currently possible. Finally, there is now clear evidence that roughly half of the halo dSphs have internal substructure. The lesson of the Sagittarius dSphs is that interactions with the Galaxy can cause distortions in the distributions of stars in the halo dwarfs. The current observational situation presents a challenge to theorists that has the potential to lead to dramatic improvements in our understanding of the role of tidal stripping in the dissolution of dwarf systems and the building of the Galactic halo.

We thank Jason Pinkney for use of his clustering statistics software and extensive consultation on its care and feeding. Ken Mighell provided us with the results for his two-dimensional Poisson statistic and with several very useful discussions and comments. P. B. E. would like to thank the Department of Physics and Astronomy at the University of Alabama and the Department of Astronomy at Ohio State University and their members for their intellectual and financial support during the course of this project. P. B. E. would also like to thank Barry Madore for pointing out what should have been the obvious. A. E. S. thanks Kyle Cudworth for the use of his proper-motion reduction software during her dissertation work, as well as the Department of Astronomy at the University of Wisconsin. This research has made extensive use of the NASA/ IPAC Extragalactic Database, which is operated by the Jet Propulsion Laboratory, California Institute of Technology, under contract with the National Aeronautics and Space Administration, and NASA's Astrophysics Data System (ADS) abstract service. The authors thank the College of Science, Engineering, and Technology of Minnesota State University for financial support of this work.

\section{REFERENCES}

Armandroff, T. E., Olszewski, E. W., \& Pryor, C. 1995, AJ, 110, 2131

Baade, W. 1950, Publ. Univ. Michigan Obs., 10, 7

Battinelli, P., \& Demers, S. 1999, AJ, 117, 1764

Bird, C. M. 1993, Ph.D. thesis, Univ. Minnesota

Cannon, R. D., \& Stobie, R. S. 1973, MNRAS, 162, 227

Cudworth, K. M., Olszewski, E. W., \& Schommer, R. A. 1986, AJ, 92, 766

Da Costa, G. S., Armandroff, T. E., Caldwell, N., \& Seitzer, P. 1996, AJ, 112,2576

Demers, S., Battinelli, P., Irwin, M. J., \& Kunkel, W. E. 1995, MNRAS, 274,491

Dressler, A., \& Shectman, S. A. 1988, AJ, 95, 985

Eskridge, P. B. 1988a, AJ, 96, 1336

Eskridge, P. B. 1988 b, AJ, 96, 1614

Fitchett, M. J., \& Webster, R. 1987, ApJ, 317, 653

Girardi, L., Bressan, A., Bertelli, G., \& Chiosi, C. 2000, A\&AS, 141, 371

Hargreaves, J. C., Gilmore, G., Irwin, M. J., \& Carter, D. 1994, MNRAS, 271,693

Harrington, R. G., \& Wilson, A. G. 1950, PASP, 62, 118

Harris, W. E. 1982, ApJS, 50, 573

Hernandez, X., Gilmore, G., \& Valls-Gabaud, D. 2000, MNRAS, 317, 831

Hesser, J. E., Harris, W. E., Vandenberg, D. A., Allwright, J. W. B., Shott, P., \& Stetson, P. B. 1987, PASP, 99, 739

Hodge, P. W. 1961, AJ, 66, 249

. 1964, AJ, 69, 438 
Irwin M., \& Hatzidimitriou, D. 1995, MNRAS, 277, 1354

King, I. 1962, AJ, 67, 471

Klessen, R. S. \& Kroupa, P. 1998, ApJ, 498, 143

Kleyna, J. T., Geller, M. J., Kenyon, S. J., Kurtz, M. J., \& Thorstensen, J. R. 1998, AJ, 115, 2359

Kroupa, P. 1997, NewA, 2, 139

Lee, K. L. 1979, J. Am. Stat. Assoc., 74, 708

Lee, M.-G., Freedman, W. L., \& Madore, B. F. 1993, ApJ, 417, 553

Lee, S.-W. 1977, A\&AS, 29, 1

Madore, B. F., \& Freedman, W. L. 1995, AJ, 109, 1645

Martínez-Delgado, D., Alonso-García, J., Aparicio, A., \& GómezFlechoso, M. A. 2001, ApJ, 549, L63

Mighell, K. J. 2001, ApJ, submitted

Mighell, K. J., \& Burke, C. J. 1999, AJ, 118, 366

Oh, K. S., Lin, D. C. N., \& Aarseth, S. J. 1995, ApJ, 442, 142
Olszewski, E. W., \& Aaronson, M. 1985, AJ, 90, 2221

Olszewski, E. W., Aaronson, M. \& Hill, J. M. 1995, AJ, 110, 2120

Piatek, S., \& Pryor, C. 1995, AJ, 109, 1071

Pinkney, J., Roettiger, K., Burns, J. O., \& Bird, C. M. 1996, ApJS, 104, 1

Schweitzer, A. E. 1996, Ph.D. thesis, Univ. Wisconsin

Schweitzer, A. E., Cudworth, K. M., \& Majewski, S. R. 1997, in ASP Conf. Ser. 127, Proper Motions and Galactic Astronomy, ed. R. M. Humphreys (San Francisco: ASP), 132

Shetrone, M. D., Côté, P., \& Sargent, W. L. W. 2001a, ApJ, 548, 592

Shetrone, M. D., Côté, P., \& Stetson, P. B. 2001b, PASP, 113, 1122

van Agt, S. L. Th. J. 1967, Bull. Astron. Inst. Netherlands, 19, 275

Walker, A. R. 1994, AJ, 108, 555

West, M. J., \& Bothun, G. D. 1990, ApJ, 350, 36

West, M. J., Oemler, A., \& Dekel, A. 1988, ApJ, 327, 1

Wilson, A. G. 1955, PASP, 67, 27 